\title{
Kinetic Pathways of Order-Disorder and Order-Order Transitions in Weakly Segregated Microstructured Systems
}

\author{
Shuyan Qi \\ Division of Physics, Mathematics and Astronomy, California Institute of Technology, Pasadena, California 91125 \\ Zhen-Gang Wang* \\ Division of Chemistry and Chemical Engineering, California Institute of Technology, Pasadena, California 91125
}

(Received 17 July 1995)

\begin{abstract}
The kinetics of hexagonal to disordered and hexagonal to body-centered-cubic phase transitions in weakly segregated, microstructured systems (e.g., diblock copolymers) is studied using a time-dependent Ginzburg-Landau (TDGL) approach. Both computer simulation of the TDGL equation and analysis of a simplified two-mode model reveal nontrivial pathways during the transition.
\end{abstract}

PACS numbers: 81.10.Aj, 64.70.-p, 81.30.Hd, 83.70.Hq

A wide variety of chemical and physical systems, such as Langmuir films, ferrofluids, and diblock copolymers, exhibit ordered periodic domain structures [1]. Irrespective of differences in the systems, the domain structures have surprisingly similar appearance: stripes and circular droplets in two dimensions, and lamellae, hexagonal (HEX) cylinders, and body-centered-cubic (bcc) spheres in three dimensions. Although the specific origins may differ from system to system, the formation of spatially periodic patterns can be attributed to the competing shortrange and long-range interactions. Near the order-disorder transition, these systems can be phenomenologically described by an order parameter free energy functional of the form

$$
F[\psi(\vec{r})]=\int d \vec{r}\left\{-\frac{\tau}{2} \psi(\vec{r})^{2}+\frac{b}{2}[\nabla \psi(\vec{r})]^{2}+\frac{v}{3} \psi(\vec{r})^{3}+\frac{u}{4} \psi(\vec{r})^{4}\right\}+\frac{c}{2} \int d \vec{r}_{1} \int d \vec{r}_{2} G\left(\vec{r}_{1}-\vec{r}_{2}\right) \psi\left(\vec{r}_{1}\right) \psi\left(\vec{r}_{2}\right),
$$

where $\psi(\vec{r})$ is the order parameter, e.g., the local magnetization in magnetic systems, or the local density contrast between the two types of monomers in diblock copolymers. $\tau$ is related to the distance from the order-disorder transition temperature, and the coefficients $b, c, u$, and $v$ are phenomenological parameters which can be computed from more microscopic models. The last term in Eq. (1) represents the long-range repulsion, which penalizes longwavelength inhomogeneities. The equilibrium properties of systems described by Eq. (1) have been the subject of extensive experimental and theoretical studies [1,2].

In this Letter, we address the phenomenology of the kinetics of the various order-order and order-disorder transitions in weakly segregated, microstructured systems, using a time-dependent Ginzburg-Landau approach. Specifically we study the kinetic pathways of HEX to disordered and HEX to bcc phases after a sudden temperature jump. This study is motivated by the general intrinsic interest in understanding kinetics of phase transitions involving spatially modulated phases, in particular, by recent experiments on diblock copolymers [3-7]. To be concrete, we shall use diblock copolymers as the context; however, we believe the phenomenology is quite general for the class of systems described by the free energy Eq. (1), and will not limit our choice of parameters specifically to those for diblock copolymers [8].

For conserved order parameters, as is appropriate for diblock copolymers, we may write the time-dependent
Ginzburg-Landau equation as [9]

$$
\frac{\partial \psi}{\partial t}=M \nabla^{2}\left(\frac{\delta F}{\delta \psi}\right)+\eta(\vec{r}, t) .
$$

Here $M$ is a mobility coefficient, which we assume to be a constant; $\eta(\vec{r}, t)$ is a random force, which for a system in equilibrium at temperature $T$, satisfies the fluctuationdissipation relation

$$
\left\langle\eta(\vec{r}, t) \eta\left(\vec{r}^{\prime}, t^{\prime}\right)\right\rangle=-2 M k_{B} T \nabla^{2} \delta\left(\vec{r}-\vec{r}^{\prime}\right) \delta\left(t-t^{\prime}\right) .
$$

As a minimal model, we ignore any hydrodynamic effects and possible nonlocality in the mobility coefficient $[10,11]$. We will also ignore the noise term in subsequent discussions except to include it as providing an initial randomness in the system. We have conducted studies that include the noise term and have confirmed that for the issues we focus on in this Letter, its effects are not crucial. It may also be commented that the distinction between conserved and nonconserved order parameter dynamics in the weak segregation limit is not essential because only a single wave number dominates in this regime. Therefore, most of our results for the case of conserved order parameters should be applicable also for nonconserved order parameters.

We first report results of numerical simulation of Eqs. (1) and (2). To facilitate the numerical procedure, we bring Eq. (2) into a cell-dynamics form suggested by Oono and co-workers $[12,13]$, with the following choice of the 
parameters: $M=1, \tau=0.24, b=0.5, c=0.02, v=$ -0.3 , and $u=0.5$. $G\left(\vec{r}_{1}-\vec{r}_{2}\right)$ is taken to be the solution of the Laplace equation, $\nabla^{2} G\left(\vec{r}_{1}-\vec{r}_{2}\right)=-\delta\left(\vec{r}_{1}-\vec{r}_{2}\right)$. The simulations are performed on a $32 \times 32 \times 32$ simple cubic lattice. A two dimensional hexagonal sinusoidal wave (in the $x-y$ plane) in the order parameter $\psi$ is first set as the initial condition with the wave vector chosen according to the minimum of the quadratic coefficient in the free energy Eq. (1). The system is then equilibrated for over 100000 steps at $\tau=0.24$. At this temperature, the system is in the stable HEX phase [see Figs. 1(a) and 1(b)] [14]. Starting from the well-equilibrated HEX phase, we change the temperature to $\tau$ values which are, respectively, in the disordered phase and in the bcc sphere phase. To start the subsequent dynamics, a small random noise is added to the perfect HEX waves. The time evolution of the system is then monitored by simulating Eq. (2) numerically. To quantify the temporal behavior of the system, we introduce a global order parameter $Q=\sum_{\vec{k}}\left|\psi_{\vec{k}}\right|^{2}$ where the $\psi_{\vec{k}}$ 's are the Fourier modes of $\psi(\vec{r})$. At the mean field level, $Q$ is zero in the disordered phase, and is positive in the ordered phases.

For a large temperature jump deeply into the disordered phase ( $\tau$ from 0.24 to 0.16 ), we find that the HEX cylinders melt after 1000 steps. The melting process appears to be a simple exponential one judged from the behavior of the order parameter $Q$. Correspondingly, a direct visual inspection of the microstructure [i.e., a gray-level plot of $\psi(\vec{r})$ in various cross sections] indicates that the melting is uniform with a monotonic decrease in the amplitude of the hexagonal waves. This behavior is consistent with the fact that at $\tau=0.16$ the system is already outside of the limit of metastability for the HEX phase [14], and therefore the amplitude of the HEX wave should follow a strictly downhill path in the free energy surface.

However, when the temperature jump is only slightly into the disordered phase $(\tau=0.24$ to $\tau=0.18)$, an interesting behavior shows up in the evolution of $Q$. A much slower decay at intermediate times is observed. At the same time, a peak in the structure factor is observed at a finite $k_{z}$, where $k_{z}$ is the wave vector along the orientation of the cylinders, indicating the formation of undulation in that direction. The undulation is seen directly in Fig. 1(c) where we plot the local order parameter $\psi(\vec{r})$ on a cut in the $y-z$ cross section. The breaking of the cylinders into droplets with a well-defined spacing seems evident. This intermediate state of modulated droplets lasts for about 1000 steps, after which the droplets melt uniformly; the decay of the order parameter $Q$ associated with this latter stage again appears to be exponential.

Although lacking perfect regularity, the microstructures shown in Fig. 1(c) are strongly reminiscent of a bcc sphere phase. This impression is supported by the fact that the bcc sphere phase lies in between the HEX phase and the disordered phase in the equilibrium phase diagram. Therefore, we have analyzed the microstructures in terms of the amplitudes of the hexagonal and bcc waves, which we denote as $A$ and $B$, respectively [15]. We observe that during the melting process, the amplitude of $B$ first increases and then decreases to zero; see Fig. 2(a).

Next we study a temperature jump into the bcc sphere phase from the HEX phase. Starting from the same equilibrated HEX, we change the parameter abruptly from $\tau=0.24$ to $\tau=0.19$. The bcc order becomes evident after 4000 steps, the spheres having grown epitaxially from the cylinders. The amplitude of $B$ increases with time to a fixed value; see also Fig. 2(b). Notice the relatively long incubation time before the appearance of the bcc waves.

Our foregoing numerical results have shown that the pathways of hexagonal to disordered and bcc phase involve the disappearance of the amplitude of the hexagonal waves and the appearance (although in some cases only transient and imperfect) of bcc waves. This observation, together with the fact that in the weak segregation limit the density modulations are nearly sinusoidal, suggests that considerable insight might be obtained by focusing on the time evolution of these dominant modes. To this end, we make the simplifying assumption that time evolution of this system can be described in terms of two order parameters $A$ and $B$ corresponding to the amplitude of the hexagonal and bcc waves at the optimal wave vector $k_{c}$ [16]. With this prescription for the representation of the modulation in the order parameter, the free energy can be written as

$$
\begin{aligned}
F[A, B]= & -3 \tau_{1}\left(A^{2}+B^{2}\right)-4 v\left(A^{3}+3 A B^{2}\right) \\
& +\frac{45 u}{2}\left(A^{4}+B^{4}+4 A^{2} B^{2}\right),
\end{aligned}
$$
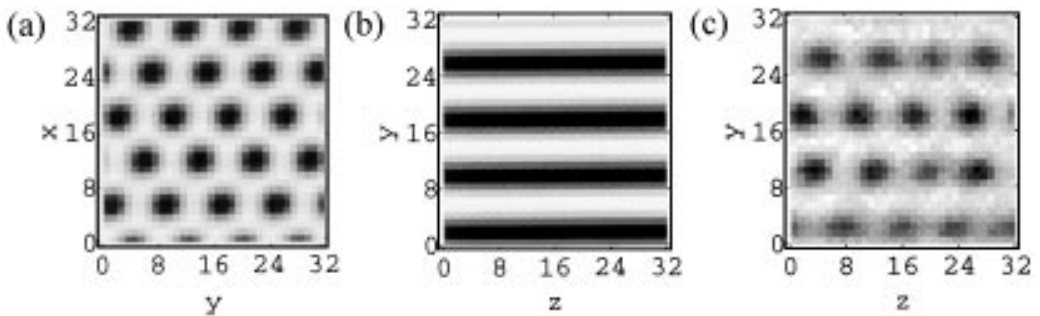

FIG. 1. Cross-sectional, gray-level view of the instantaneous configurations from the simulation. (a) $x$ - $y$ cross section at $t=0$; (b) $y-z$ cross section at $t=0$; (c) $y-z$ cross section at $t=1000$ steps. The dark regions are for $\psi>0$, and the light regions are for $\psi<0$. 

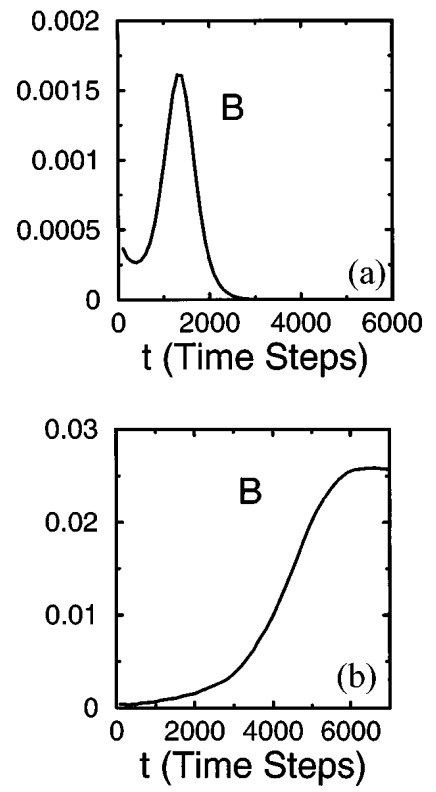

FIG. 2. Time evolution of the amplitude of bcc waves $B$ (a) for a temperature jump slightly into the disordered phase (from $\tau=0.24$ to $\tau=0.18$ ), and (b) for a temperature jump into the bcc phase (from $\tau=0.24$ to $\tau=0.19$ ). The relatively large initial $B$ in (a) is due to the initial noise that is included in the simulation.

where $\tau_{1}=\tau-2 \sqrt{b c}$. Correspondingly, the dynamic equation (2) becomes [16]

$$
\begin{gathered}
\frac{\partial A}{\partial t}=-M k_{c}^{2}\left[-A \tau_{1}-2 v\left(A^{2}+B^{2}\right)\right. \\
\left.+15 u\left(A^{3}+2 A B^{2}\right)\right], \\
\frac{\partial B}{\partial t}=-M k_{c}^{2}\left[-B \tau_{1}-4 v A B+15 u\left(B^{3}+2 B A^{2}\right)\right] .
\end{gathered}
$$

Note that if we set $B=0$, the stationary solution of Eq. (5) at the temperature $\tau_{1}$ yields the equilibrium value for the order parameter $A_{0}$ of the HEX phase in the single wave number approximation. The stability of this solution after a temperature jump to $\tau_{1}^{\prime}$ can be analyzed by performing a linear stability analysis. If $\tau_{1}^{\prime}$ lies within the spinodal of the hexagonal phase, there is no linear instability. Our calculated phase diagram shows that the spinodal temperature of $A$ is within the bcc phase region, thus temperature jumps to the disordered phase are already outside the metastable region. Therefore, there will always be an initial driving force after the temperature jump for $A$ to decay. On the other hand, the behavior of $B$ in Eq. (6) is different depending on the net coefficient of the linear term on the right-hand side. If the coefficient is negative, then any small perturbation on $B=0$ will decay exponentially. However, if the coefficient is positive, which occurs when $A$ is negative (this ensures the epitaxial relationship between the cylinders and the spheres that grow out of them) and within a certain bracket and when the temperature jump is small, a perturbation from
$B=0$ will initially grow. This is the scenario for the transient appearance of the bcc structure during the melting of the hexagonal structure for a slight temperature jump to the disordered phase. Our calculation shows that if the temperature jump is within some window, the melting of the cylinder will go through a transient bcc phase. In the mean-field phase diagram, this window is bounded by the order-disorder boundary, which is given by $\tau_{1}=-32 v^{2} / 405 u$, and the curve $\tau_{1}=-2 v^{2} / 15 u$.

We show the phase portrait in terms of $A$ and $B$ for the slight temperature jump from the HEX phase to the disordered phase in Fig. 3(a). The arrow indicates the direction of time progression. The trajectory clearly shows the appearance of a bcc wave during the melting of the HEX cylinders.

In Fig. 3(b) we show the time evolution of the order parameters $-A$ and $B$ after a temperature jump to the bcc phase. It is seen that while $A$ has a rapid initial decay, the growth of $B$ becomes appreciable only after 100 time units. This is consistent with the result from our simulation. Another interesting feature is that there is a long plateau region for $A$ in the intermediate times. In fact, between 50 and 100 time units, there is little change in either $A$ and $B$, suggesting that the system has come near to a saddle point.

Further insight into the time evolution of the microstructures after a temperature jump is obtained by considering the free energy surface in the order parameters $A$ and $B$. This is possible because in the single wave number approximation, the Laplacian operator in Eq. (1) is transformed to $-k_{c}^{2}$ [16], a constant factor. Thus, Eqs. (5) and (6) simply describe the steepest descent of the order
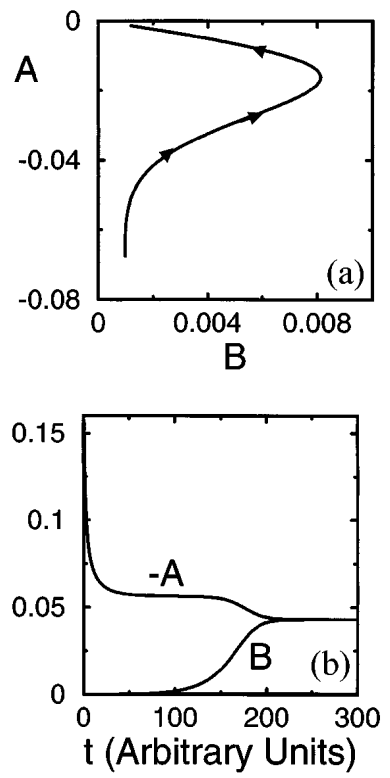

FIG. 3. Results from analyses using Eqs. (5) and (6): (a) Phase portrait of $B$ vs $A$ for a temperature jump slightly into the disordered phase (from $\tau=0.24$ to $\tau=0.185$ ). The nonzero initial $B$ is due to the initial perturbation; (b) time evolution of the order parameters $A$ and $B$ after a temperature jump into the bcc phase. 
(a)

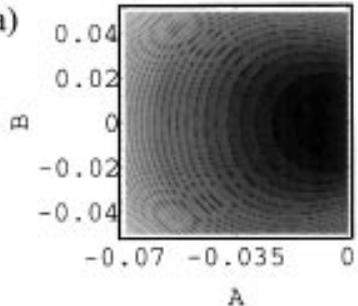

(b)

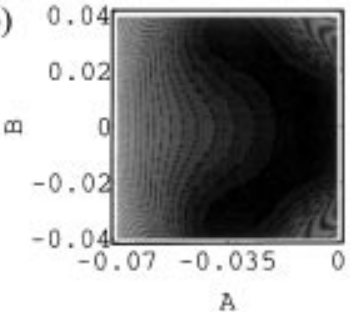

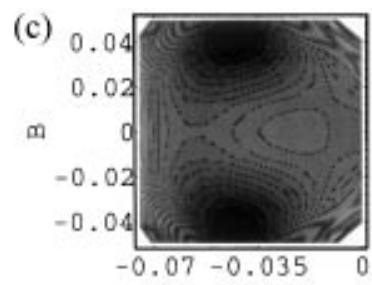

A

FIG. 4. Free energy contour plots in the order parameter space $A$ - $B$ : (a) deeply in the disordered phase $(\tau=0.16)$, (b) slightly in the disordered phase $(\tau=0.185)$, and (c) in the bcc phase $(\tau=0.19)$.

parameters $(A, B)$ on the free energy surface. The dynamics can then be understood by following the path of the largest downhill gradient in the free energy landscape. Figure 4 shows contour plots of the free energy in the parameter space $A, B$ for the three situations we have studied. It is clear that for a jump from the hexagonal phase deeply into the disordered phase, the steepest path is along the $B=0$ axis, with the global minimum at $A=0$ and $B=0$, indicating a direct melting of the cylinders without the appearance of the droplets. On the other hand, for a shallow temperature jump, while the line along $B=0$ is still in a downhill direction, this line is actually a ridge, and therefore a small perturbation with a nonzero $B$ will lead to a transient growth in $B$ from this path before the system settles in the global minimum $A=0$ and $B=0$. Finally, for a temperature jump into the bcc phase, the global minimum is now at $A=-0.043$ and $B=0.043$, and a saddle point exists at $A=-0.056$ and $B=0$. The steepest path can get rather close to this point, which is the reason for the near stagnation in the time evolution of both order parameters.

The epitaxial relationship between the cubic phases and the HEX phase in diblock copolymers has been established experimentally by Bates et al. [5-7]. These authors also reported observation of transient sphere structures during the melting of the HEX in shear-cessation experiments [6,7]. In one of these experiments, an initially disordered phase of asymmetrical poly(ethylenepropylene)-poly(ethylethylene) close to the order-disorder phase boundary is subjected to a constant shear which induces a transition to the HEX phase (with the cylinders aligned along the shear direction). Then the shear is suddenly stopped, and the system is now in a condition favoring the disordered phase. These authors observed transient sphere structures which grow epitaxially from the cylinders by means of neutron scattering. They attribute the transient epitaxial appearance of the spheres to the anisotropic suppression (during shear) and reemergence (after shear is stopped) of thermal fluctuations. Although shear flow is involved in the experiment, it is our opinion that the essence of the experiment is to prepare a cylinder phase and then suddenly change the conditions to favoring the disordered phase. In this regard, the shear-cessation experiment can be likened to the temperature jump studied in this Letter. The dominant mechanism for the appearance of the transient structure could well be that depicted here.
We thank S. T. Milner, G. H. Fredrickson, and P.-G. de Gennes for helpful discussions. This research is supported in part by donors of the Petroleum Research Fund, administered by the American Chemical Society, and by the National Science Foundation (Grant No. ASC9217368).

*Author to whom correspondence should be addressed.

[1] M. Seul and D. Andelman, Science 267, 476 (1995), and references therein.

[2] See F. S. Bates, Science 251, 898 (1991); F. S. Bates and G. H. Fredrickson, Ann. Rev. Phys. Chem. 41, 525 (1990), and references therein.

[3] S. Sakurai et al., Macromolecules 26, 485 (1993).

[4] D. A. Hajduk et al., Macromolecules 27, 490 (1994).

[5] M. F. Schulz et al., Phys. Rev. Lett. 73, 86 (1994).

[6] K. A. Koppi, Ph.D. thesis, The University of Minnesota, 1994.

[7] F. S. Bates, K. A. Koppi, and M. Tirrell, Macromolecules 27, 5934 (1994).

[8] For diblock copolymers, Eq. (1) can be derived by following the RPA approach of Leibler and making further simplifications on the wave number dependence of the various vertex functions. See L. Leibler, Macromolecules 13, 1602 (1980); T. Ohta and K. Kawasaki, ibid. 19, 2621 (1986); A. M. Mayes, M. O. de la Cruz, and W.E. McMullen, ibid. 26, 4050 (1993).

[9] J. D. Gunton, M.S. Miguel, and P.S. Sahni, Phase Transition and Critical Phenomena, edited by C. Domb and J. L. Lebowitz (Academic Press, New York, 1983), Vol. 8.

[10] H. P. Wittmann and G. H. Fredrickson, J. Phys. I (France) 4, 1791 (1994).

[11] P.-G. deGennes, J. Chem. Phys. 72, 4756 (1980); P. Pincus, ibid. 75, 1996 (1981); K. Binder, ibid. 79, 6387 (1983).

[12] S. Puri and Y. Oono, Phys. Rev. A 38, 1542 (1988).

[13] M. Bahiana and Y. Oono, Phys. Rev. A 41, 6763 (1990).

[14] We have calculated the mean-field phase diagram as well as the various spinodals.

[15] When the axis of cylinder is along $(1,1,1)$ of a cubic lattice, $A$ denotes the magnitude of waves along $(-1,1,0)$, $(0,-1,1)$, and $(-1,0,1)$, and $B$ denotes the magnitude of waves along $(1,1,0),(0,1,1)$, and $(1,0,1)$.

[16] The dominant wave vector determined from the dynamic equation is slightly different from that determined from the free energy. However, consistent with the single wavelength approximation, we ignore this difference. 